\title{
Suppository Solid Provision Technology
}

\author{
Samuel Iwobi \\ Department of Chemistry, Collage of Science and Technology, \\ Covenant University, Nigeria
}

\begin{abstract}
Suppository can be used for systemic effects in conditions where oral drug preparations will not be resistant or absorbed rapidly. Once inserted the base suppository melts, softens or dissolves causing the underlying medicinal substance to enter the tissues of the area. In the manufacture of suppositories, there is a term known as the exchange rate for making oleum cacao based. Therefore, weighing should not be carried out one by one, but the exchange rate of active substances is calculated to find the required oleum cacao. The advantage of weighing the ingredients is to provide the suppository period at the time of printing.
\end{abstract}

Keywords: Suppository, Melting, Oleum

Received : August 17, 2020

Received in Revised: August 25, 2020

Accepted: August 28, 2020

\section{Introduction}

Suppositories are a solid dosage form that is used by inserting them through a hole or gap in the body, where it will expand, soften, or dissolve and give local and systemic effects (Hermann, 1995).

The rectal suppository weighs about 2 grams for adults and is usually oblong like a torpedo. Suppositories for children weigh about 1 gram and are smaller in size. The vaginal suppository is approximately 3-50 grams and is round or ovoid in shape. The urethral suppository is pencil-shaped and tapered at one end, the urethral suppository used for men weighs approximately 4 grams and is 60-75 mm long (Allen, 1997).

The advantage of suppositories, i.e. drugs that are destroyed or rendered inactive by $\mathrm{pH}$ or enzyme activity of the stomach or intestines, need not be introduced into this destructive environment, drugs that damage or stimulate the stomach can be administered without stimulation, drugs that are damaged in the portal circulation, can pass through the liver after absorption into the rectum (Liang \& Liang, 2002). In oral use, this method is more suitable for use by adult patients and children who cannot or do not want to swallow the drug, and is an effective way of treating patients who like vomiting.

The advantage of suppositories is that they can be used for systemic effects in conditions where oral drug preparation will not be resistant or absorbed quickly, such as in a state of intense nausea or in a state of unconsciousness (Hooker et al, 1996).

Suppository bases have an important role in releasing the drugs they contain. One of the main requirements of a suppository base is that it is always solid at room temperature but immediately softens, fuses or dissolves at body temperature so that the drug it contains can be fully available after use (de Blaey \& Fokkens, 1985).

The base has reached a balance of constancy where the components melt at a rectal temperature of $36 \mathrm{C}$, are not toxic and do not irritate sensitive and inflamed tissues, can mix with various drugs, the base suppository does not have a stable meta (does not change in its original state at the time of melting), The suppository base shrinks sufficiently upon cooling, the suppository base has wetting and emulsifying properties, the suppository base 
does not stimulate, the high water number means a high percentage of water can be intended into it, has no medicinal effect, can be made suppositories by hand, compression or abstruction machines.

\section{Therapeutic effect on suppositories}

\section{Local action}

Once inserted the base suppository melts, softens or dissolves causing the underlying medicinal substance to enter the tissues of the area. This drug is intended to be held in these spaces for local or systemic action. Rectal suppositories are intended for local action and are most often used to relieve constipation and pain, irritation, itching, and inflammation associated with hemorrhoids/other anarectal conditions.

\section{Systemic action}

Medicines can be given in the form of suppositories for both local and systemic effects (Liang \& Liang, 2002). This action depends on the effect of the drug, its concentration and rate of absorption of emollients, astringents, antibacterial agents, hormones, steroids and local anesthetics given in the form of suppositories to treat local conditions of the vagina, rectum, or urethra.

Suppositories can act as local tissue protectors as carriers of eutic therapeutic agents as local effects or systemic effects of the base suppositories used greatly affect the release of therapeutic agents. Hemorrhoids are divided into two; namely internal hemorrhoids and external hemorrhoids. Internal hemorrhoids are located proximal to the lines paltinea and are covered by the epithelial layer of the mucosa which is a lump of internal hemorrhoidal veins. Meanwhile, external hemorrhoids occur in the swelling of the external hemorrhoidal plensus, located distal to the linea pactinea and covered by the tissue under the anal epithelium (Yahata et al, 2002).

The circulation pathway in the rectum plays the most important role in the effect produced by this route of use, the supply of rectal blood by the three veins namely upper, lower and middle hemorrhoids (Santos et al, 2013). The drug is absorbed through the rectal bile lining and enters these three veins to distribute throughout the body because the lower and middle hemovoral veins are connected to the interior kappa vein, so most of the drugs that are absorbed through these two veins do not pass through the liver which causes avoidance of the process of drug substance and from the liver about 50/70\% of the drug substance absorbed from the rectal suppository enters through the general circulation and passes through these two veins while the top hemorrhoid vein in another position is through the portal system of the liver so that the drug material is absorbed by these blood vessels and enters through the liver before being spread into the blood circulation, the distribution process of drug distribution and absorption through the rectum.

\section{Factors affecting absorption of drug suppositories}

\section{Physiological Factors}

A number of drugs cannot be given orally, because they are affected by digestive juices or their therapeutic activity is altered by the liver after absorption. After the drug is absorbed from the soft intestine, it will be lowered by the hepatic vein to the liver, the liver changes most of the drug chemically so that its systemic effectiveness is often reduced.

The $\mathrm{pH}$ of the rectal mucosa has a role in controlling the rate of drug absorption, which means that the rectal fluid does not actually have a buffering capacity, and this results in the dissolved drug determining the $\mathrm{pH}$ in the anorectal area. So the absorption of a drug will increase by changes in the $\mathrm{pH}$ of the rectal mucosa and will increase the ratio that is not 
ionized. The effect of $\mathrm{pH}$ in the lumen on the absorption of several acidic drugs and alkaline drugs.

\section{Physic-chemical factors}

In order for the drug to be absorbed, it must be removed from the suppository and distributed by the surrounding fluid at the absorption sites by dissolving in the liquid, there will be extensive contact of the drug with the lumen wall, thereby increasing drug contact with most absorption places. The coefficient of water fat tends to dissolve in fat, the drug is slowly released from the suppository carrier.

\section{Methods}

The tools used in this experiment were: aluminum foil, stirring rod, porcelain cup, suppository mold, beaker glass, parchment paper, water bath, horn spoon, thermoeter, analytical scale. The materials used in this experiment were: Alpha Tocopherol, Aspirin, Cera alba, Glycerin and oleum cacao.

\section{Basic Formula}

Suppositories are a solid dosage form that is used by inserting through a hole or gap in the body, where it will melt, soften or dissolve and give a local or systemic effect. Suppositories are generally inserted through the rectum, vagina and sometimes through the urinary tract and rarely through the ears and nose. The shape and size must be such that it can easily be inserted into the desired hole or crevice without causing irregularities and the inflating must last for a period of time.

The drugs are given in the form of suppositories with local effect or systemic effect, the action depends on the nature of the drug, its concentration and absorption rate, astrigen, antibacterial substances, steroid hormones and anesthetics of the vagina, rectum and urethra. Its main purpose for use of rectal suppositories is also given for systemic effects in which the oral administration of the drug will not be analyzed and absorbed properly as in a normal state free from vomiting or on an oblique position.

The pour method is the method most commonly used to make small-scale and large-scale suppositories. The first process of molding the base is melted to avoid solidification then the active ingredient is emulsified or absorbed therein and poured into a metal mold coated with nickel.

The advantage of drug suppositories that are damaged or rendered inactive by $\mathrm{pH}$ or enzyme activity of the stomach or intestines does not need to be brought into this luxurious environment, drugs that damage or stimulate the stomach can be administered without stimulation, drugs that damage the portal circulation can pass through the liver after absorption in the rectum for oral use, this method is more suitable for use by adult patients and children who are unable or unwilling to take medication and is an effective way of treating patients who vomit.

The reason for choosing the concentration of the active substance is that aspirin can be administered rectally with suppositories. For a good anti-inflammatory effect, the plasma level needs to be maintained between $250-500 \mathrm{mg} / \mathrm{ml}$.

\section{Substitute Calculation}

Suppo weight only base: 1.81 grams

The weight of the supply base only $+10 \%$ of the active substance: 1.91 grams 
The number of active substances in suppo (b): $\frac{10}{100} \times 1,59$ gram : 0,191 gram

Number of bases in suppo (b) $\quad:(1,91$ gram $-0,191$ gram $)$

$$
: 1,71 \text { gram }
$$

Comparable to base on suppo (a) : (1,81 gram - 0,171 gram)

$$
\text { : } 0,1 \text { gram }
$$

So, 0.1 gram base \pm 0.191 gram of aspirin

Or 1 gram of aspirin $\approx 0.51$ gram of base

\section{Suppo Weight Calculation}

Suppo Weight

: 2,122 gram

Aspirin

: 0,65 gram

Base

:2,122 gram $-0,65$ gram : 1,472 gram

Aspirin

$: 0,65$ gram $\approx(0,65$ gram $\times 0,52$ gram $)$ base

: 0,388 gram

Suppo ideal weight $\approx$ just bases only : 1,81 gram

The base added is

$$
: 1,81 \text { gram }-0,388 \text { gram }
$$

$$
\text { : } 1,472 \text { gram }
$$

Actual support weight $\quad: 0,65 \mathrm{~g}+1,472 \mathrm{~g}: 2,122 \mathrm{~g}$

\section{For 15 suppo, weigh the ingredients a number}

Aspirin Weight

: 0,65 gram $\times 15$ suppo $: 9,75$ gram

Base ideal weight: 1,472 gram $\times 15$ suppo : 22,08 gram

Cera alba weight

$$
: \frac{3 \mathrm{gram}}{100 \mathrm{gram}} \times 22,08 \text { gram }: 0,66 \text { gram }
$$

Oleum cacao weight $\quad: 22,08$ gram $-0,66$ gram

$$
\text { : 21,42 gram - 0,085 gram : 21,33 }
$$

Alpha tocopherol weight : $\frac{0,05 \mathrm{gram}}{100 \mathrm{gram}} \times 2,122 \mathrm{~g}$

$$
\text { : 0,00106 g× } 15 \text { suppo : 0,015 g : 15,9 mg }
$$

Alpha tocopherol dilution

$1 \mathrm{mg}$ alpha tocopherol $\approx 1,49 \mathrm{UI}$

$1 \mathrm{UI}: \frac{1}{1,49} \mathrm{mg}: 0,6711 \mathrm{mg}$

$100 \mathrm{UI}: 0,6711 \mathrm{mg} \times 100$

$$
\text { : } 67,11 \mathrm{mg}
$$

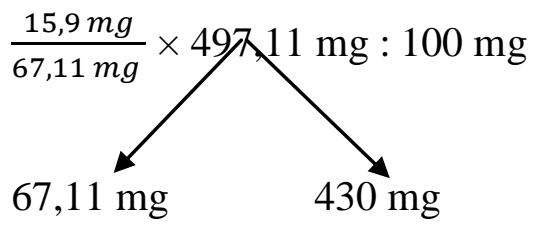


(Alpha tocopherol 1 capsule) (Oleum cacao)

\section{Discussion}

The suppository preparations made in this practicum use aspirin as an analgesic and antipyretic. The reason aspirin is used is because salicylic acid as an antipyretic used as an external drug, its derivatives that can be achieved systemically are salicylic esters and organic acids with substances in the hydroxy group such as acetosal, so the substance used is acetosal (de Vries \& ten Cate, 1969). Suppositories have several advantages, namely that they can prevent drug damage by digestive enzymes and stomach acid, both for patients who vomit easily or are unconscious (Ham \& Buckheit Jr, 2017). Systemic action is often used as an absorption site, drugs that are used through the rectum in the form of suppositories to obtain systemic effects consisting of aspirin for analgesic and antipyretic activity.

The method used in the manufacture of suppositories is cast printing because the method most commonly used to make suppositories is suitable for all types of bases and can be used for small and large scales.

In the manufacture of suppositories, there is a term known as the exchange rate for making oleum cacao based. The exchange rate is intended to determine the weight of brown fat which has the same volume as 1 gram of drug. Therefore, weighing should not be carried out one by one, but the exchange rate of active substances is calculated to find the required oleum cacao. The advantage of weighing the ingredients is to provide the suppository period at the time of printing. When filling the suppository into the mold, the brown fat quickly freezes, and during cooling, the volume shrinks until the hole occurs above the mass, so the mold filling must be filled more, only after chilling the excess.

In the first experiment, a clean and dry suppository mold was prepared, and glycerin was added to the destination mold, added with glycerin, which is as a lubricant so that the material does not stick to the mold, then the mold is closed so that there is no accumulation of glycerin in the mold, melted with cera alba and some oleum cacao in it. a porcelain cup over a water bath at $37 \mathrm{oC}$ so that the suppository can melt at body temperature and then stir evenly. Add the remaining oleum cacao to the porcelain cup slowly by paying attention to the consistency of the melt and stirring evenly, then removing the base melt from the water bath and adding the aspirin powder slowly and stirring until it is homogeneous (mixing the base with the active substance should be done quickly so as not to cool down during the process. mixing the active substance with the base), filled with melt into the mold (the mold temperature should be the same as the melt) with the help of a stirring rod, allowed the mixture to solidify at room temperature, for about 15 minutes the goal is to let stand 15 minutes so that the supply does not suffer damage, put the mixture into refrigerator (temperature $8-10 \mathrm{oC}$ ) for 10 minutes, then put in the freezer for 5 minutes, after which the excess mass that has solidified is cut, removed the suppository from the mold and evaluated the suppository according to official requirements and industry requirements.

\section{Conclusion}

Based on the experimental results, it can be concluded that the method of determining the replacement number is by using two prints. The first mold is only the base and the second mold is added with the active substance. The method used in making suppositories is the cast molding method because the most common method used to make suppositories is suitable for all types of bases and can be used for small and large scales.

\section{References}

Allen, L. V. (1997). Suppositories as drug delivery systems. Journal of Pharmaceutical Care in Pain \& Symptom Control, 5(2), 17-26. 
de Blaey, C. J., \& Fokkens, J. G. (1985). Drug release from suppositories. Pharmaceutical research, 2(2), 61-64.

de Vries, S. I., \& ten Cate, J. W. (1969). Thrombopathy following use of Acetosal. Nederlands tijdschrift voor geneeskunde, 113(17), 752.

Ham, A. S., \& Buckheit Jr, R. W. (2017). Designing and developing suppository formulations for anti-HIV drug delivery. Therapeutic delivery, 8(9), 805-817.

Hermann, T. W. (1995). Recent research on bioavailability of drugs from suppositories. International journal of pharmaceutics, 123(1), 1-11.

Hooker, G. D., Gregor, J. C., Ponich, T. P., \& McLarty, T. D. (1996). Diaphragm-like strictures of the right colon induced by indomethacin suppositories: evidence of a systemic effect. Gastrointestinal endoscopy, 44(2), 199-202.

Liang, K., \& Liang, L. (2002). U.S. Patent Application No. 09/974,452.

Santos, I. S., Ponte, B. M., Boonme, P., Silva, A. M., \& Souto, E. B. (2013). Nanoencapsulation of polyphenols for protective effect against colon-rectal cancer. Biotechnology advances, 31(5), 514-523.

Yahata, T., Takedatsu, H., Dunwoodie, S. L., Bragança, J., Swingler, T., Withington, S. L., ... \& Shioda, T. (2002). Cloning of mouse Cited4, a member of the CITED family p300/CBP-binding transcriptional coactivators: induced expression in mammary epithelial cells. Genomics, 80(6), 601-613. 